\title{
Evaluation of the Antioxidant Activity of Three Different Brown Macroalgae by Implementing Electrochemistry and Spectrophotometry: Correlation and Prospects
}

\author{
Čižmek Lara', Martić Ana ${ }^{1}$, Čož-Rakovac Rozelindra', Trebše Polonca ${ }^{2}, *$ \\ 1. Laboratory for Aquaculture Biotechnology, Division of Materials Chemistry, Ruđer Bošković Institute, Bijenička 54, 10000 Zagreb, Croatia \\ 2. University of Ljubljana, Faculty of Health Sciences, Zdravstvena pot 5, 1000 Ljubljana, Slovenia \\ * Correspondence: Polonca Trebše; polonca.trebse@zf.uni-lj.si;
}

Citation: Čižmek L, Martić A, Čož-Rakovac R, Trebše P. Evaluation of antioxidant activity of three different brown macroalgae by implementing electrochemistry and spectrophotometry: Correlation and future prospects. Proceedings of Socratic Lectures. 2021; 6: 38-47. https://doi.org/10.55295/PSL.2021.D. 006

Publisher's Note: UL ZF stays neutral with regard to jurisdictional claims in published maps and institutional affiliations.

Copyright: $(C) 2021$ by the authors. Submitted for possible open access publication under the terms and conditions of the Creative Commons Attribution (CC BY) license (https://creativecommons.org/licenses/b $\mathrm{y} / 4.0 /)$.

\begin{abstract}
:
In this study, a comprehensive evaluation of the antioxidant activity of three brown macroalgae Dictyota dichotoma, Dictyota fasciola, and Halopteris scoparia from the Adriatic Sea (Croatia) by implementing electrochemical and spectrophotometrical measurements was performed. The aim was to find out if the used methods correlate with each other to obtain information about the present antioxidant activity. The samples were subjected to fractionation by solid-phase extraction (SPE) which allowed the separation of different polarity compounds. Voltammetry of microfilm immobilized on a glassy carbon electrode using square-wave voltammetry (SWV) was applied as an electrochemical method, and the obtained results were correlated to the results of four spectrophotometric methods, namely the reduction of the radical cation (ABTS), the 2,2-diphenyl-1-picryl-hydrazyl (DPPH) assay, the Folin-Ciocalteu, and the oxygen radical absorbance capacity (ORAC) assay. All methanolic fractions from three brown macroalgae showed higher antioxidant activity than the dichloromethane fractions. The highest activity was obtained for $H$. scoparia methanolic fraction with an $\mathrm{IC}_{50}$ concentration of $1.947 \mathrm{mg} / \mathrm{mL}$. It was found that antioxidant activity obtained by SWV only correlates well to 1,1-diphenyl-2-picrylhidrazine (DPPH) radical assay $\left(R^{2}=0.83, p<0.05\right)$, while a good correlation was found between ABTS and ORAC assays $\left(R^{2}=0.0 .94, p<0.05\right)$. Correlations indicate that the synergistic effect of different compounds extracted from the samples impacted their antioxidant response. All three researched brown macroalgae have shown to be a potent source of natural antioxidants that could further be used in the research of oxidative stress-related diseases.
\end{abstract}

Keywords: Brown seaweeds; Marine organisms; Voltammetry; Extraction; Bioactivity 


\section{Introduction}

Marine macroalgae or seaweeds are in the last decade in a continuous focus of the scientific community, but even in the focus of a wider public as they are acknowledged as a valuable source of different bioactive compounds such as polyphenols, peptides, pigments, and polysaccharides that are associated with several health benefits and biological activities (Afonso et al., 2019; Cardoso et al., 2014; Cikoš et al., 2021). Evolutionary development of such a variety of natural compounds was enhanced by macroalgal adaptation to extreme marine conditions in terms of temperature, light, pressure, oxygen, salinity, as well as microbial and viral attacks, and toxicity (Ruocco et al., 2016; Zhao et al., 2018). Thus, the chemical composition of macroalgae varies considerably not only among different species but also within the same species that inhabited different ecosystems.

Brown algae are known for their accumulation of specific metabolites with great antioxidant potential, especially phenolic compounds phlorotannins, polysaccharides alginates, laminarans, and fucoidans with antiviral, anti-inflamatory, antitumoral, antithrombotic, and antioxidant activity (Rabanal et al., 2014) along with carotenoid fucoxanthin with antiproliferative effects on cancer cells (Afonso et al., 2019; Ktari et al., 2021). Brown algae Dictyota dichotoma and Dictyota fasciola belong to the Dictyotaceae family known for producing polyphenols, diterpenes, and sulfated polysaccharides, especially during stress conditions possessing the capacity of protecting the immune system and serving as scavengers of oxygen free radicals (Sathya et al., 2017), while the extracts of Halopteris scoparia have shown the potential to be used as a protective and preventive agent against human cancers (Güner et al., 2019).

Antioxidants are a highly heterogeneous group of chemical compounds, and many methods have been developed to evaluate the antioxidant potential of algae biomass extracts (Kohen et al., 2002; Prior et al., 2005). Most assays for the determination of antioxidant activity are based on chemical reactions of antioxidants with a probe, which has a different color depending on its redox state, followed by spectrophotometric measurement. The main disadvantage of this type of methods is that they are based on color formation, which can be problematic when applied to algal extracts due to high concentrations of colored chlorophylls and carotenoids, so a blank solution has to be measured for each tested sample and concentration. Also, the chemical assays measure only a fraction of the total antioxidant activity in the extract as the performed redox reactions have a specific redox potential (Goiris et al., 2012). Electrochemical (voltammetric) methods have been recognized as an alternative to chemical antioxidant assays (Čižmek et al., 2021; Goiris et al., 2012). Some of the benefits of voltammetric measurements are sensitivity (analysis of both lipophilic and hydrophilic extracts, and can be carried out on colored samples), rapidity, simplicity (no need for laborious sample preparation and simple equipment), and the capacity to directly measure the number of electrons transferred by an antioxidant. The obtained peak potentials detected in the voltammogram can be used as an indicator of the redox potential of major antioxidants present in the sample and their reducing power. Voltammetry provides quantitative information on the total antioxidant capacity of the sample given by the total area under the curve (AUC). This is especially important when crude samples are measured, in which usually several compounds with various oxidation potentials contribute to the AUC (Goiris et al., 2012; Haque et al., 2021).

In this study, we evaluated the antioxidant activity of two different fractions from three brown macroalgae. The primarily used method was square-wave voltammetry (SWV), followed by the reduction of the radical cation (ABTS), the 2,2-diphenyl-1-picryl-hydrazyl (DPPH) assay, the Folin-Ciocalteu, and the oxygen radical absorbance capacity (ORAC) assays. To correlate obtained results for antioxidant activity, Pearson's correlation coefficient was used. 


\section{Material and Methods}

\subsection{Chemicals}

The standards of gallic acid (>97.5\%), L-ascorbic acid ( $\geq 99 \%)$, DPPH (2,2-diphenyl-1picrylhydrazyl), ABTS (diammonium salt of 2,2'-azino-bis(3-ethylbenzthiazolin-6-yl) sulfonic acid, $>99.0 \%$ ), dichloro-dihydro-fluorescein diacetate ( $\geq 97 \%$, DCF-DA), AAPH (2,2-azobis (2-methylpropionamidine) dihydrochloride, 97\%) and 2', $7^{\prime}$-dichloro fluorescin diacetate were purchased from Sigma-Aldrich (St. Louis, MO, USA).

Dimethyl sulfoxide (DMSO, p.a.), methanol (p.a.), ethanol (p.a.), hydrochloric acid ( $\mathrm{HCl}$, p.a.), sodium carbonate $\left(\mathrm{Na}_{2} \mathrm{CO}_{3}\right.$, p.a.), dibasic sodium phosphate $\left(\mathrm{Na}_{2} \mathrm{HPO}_{4}{ }^{*} 2 \mathrm{H}_{2} \mathrm{O}\right.$, p.a.), monobasic sodium phosphate $\left(\mathrm{NaH}_{2} \mathrm{PO}_{4}{ }^{*} \mathrm{H}_{2} \mathrm{O}\right.$, p.a.) and Folin-Ciocalteu reagent were obtained from Kemika (Zagreb, Croatia) while potassium persulfate $(>98 \%)$ was purchased from Scharlau, (Regensburg, Germany).

The solvents used for solid-phase extraction were of HPLC grade and were obtained from Honeywell (Charlotte, NC, USA).

\subsection{Macroalgae samples and extraction procedure}

Three brown marine macroalgae: Dictyota dichotoma (DIDI), Dictyota fasciola (DIFA), and Halopteris scoparia (HASC) were collected from the Adriatic Sea, transported, cleaned with distilled water several times, freeze-dried, and stored at $4{ }^{\circ} \mathrm{C}$ until further analysis. The extraction was performed on $5 \mathrm{~g}$ of each macroalgae sample with a mixture of solvents methanol $(\mathrm{MeOH}) /$ dichloromethane $(\mathrm{DCM})(1: 1, v / v)$, in an ultrasonic bath (Bandelin, Sonorex digiplus 560W, Germany) for $5 \mathrm{~min}$. The extraction of each sample was repeated three times with centrifugation between each sonication. The supernatants were collected and mixed with C18 powder and then subjected to evaporation under nitrogen flow (5.0, Messer, Croatia). The SPE cartridges (C18, Agilent Bond Elut, Germany) were conditioned with methanol and ultrapure water, and afterward, the dry extracts were eluted using solvents with decreasing polarity (water $>\mathrm{MeOH}>\mathrm{DCM}$ ). Polar fraction F1 was discarded and was not analyzed within this study, while less polar fractions (F2 and F3) were collected and dried under nitrogen flow. Before the analysis, the fractions were dissolved with appropriate solvents, $\mathrm{F} 2$ fraction in $\mathrm{MeOH}$ and F3 fraction in DCM.

\subsection{Electrochemical analysis}

The electrochemical experiments were carried out in accordance with our recently published paper (Čižmek et al., 2021). Briefly, a computer-controlled PalmSens electrochemical system (Houten, the Netherlands) with PSTrace software was used with glassy carbon electrode (GC electrode, $\mathrm{BASi}$, diameter $3 \mathrm{~mm}$ ) as a working electrode, an $\mathrm{Ag} / \mathrm{AgCl}(3 \mathrm{~mol} / \mathrm{L} \mathrm{KCl})$ electrode as a reference electrode and a platinum wire as a counter electrode. All potentials were expressed versus $\mathrm{Ag} / \mathrm{AgCl}(3 \mathrm{~mol} / \mathrm{L} \mathrm{KCl})$ reference electrode. Cyclic (CV) and square-wave (SWV) voltammetry were performed on all samples in $0.1 \mathrm{~mol} / \mathrm{L}$ phosphate buffer solution (PBS) as an electrolyte solution. Analysis of samples was performed by pipetting the known volume of obtained sample $(5 \mu \mathrm{L})$ on the surface of the GC working electrode and left to dry at room temperature for a minute. Unless otherwise stated, all measurements were performed in triplicates. CV experiments on modified GC electrode were performed at a potential scan rate of $50 \mathrm{mV} / \mathrm{s}$, while SWV was performed using a potential step increment of $2 \mathrm{mV}$ and a square-wave amplitude of $20 \mathrm{mV}$. The frequency varied from 10 to $200 \mathrm{~Hz}$. The change in $\mathrm{pH}$ value was also analyzed.

\subsection{Spectrophotometric analysis}

Antioxidant activity was evaluated by implementing four in vitro assays: reduction of radical cation assay (ABTS), 2,2-diphenyl-1-picrylhydrazyl-hydrate (DPPH) assay, oxygen radical absorbance capacity (ORAC) assay, and Folin-Ciocalteu method. All measurements were performed in triplicates on a spectrophotofluorimeter microplate reader (Infinite M200 PRO, TECAN, Switzerland) in the multi-well plate (96-well). By the 
reactions with appropriate reagents, obtained fractions were tested for their antioxidant activity. Their color change, with regards to control or blank sample, was measured. The F2 and F3 fractions of each macroalga were prepared at a stock concentration of 10 $\mathrm{mg} / \mathrm{mL}$ and appropriate dilutions of each sample were prepared for further analysis. A detailed description of all preformed methods can be found in recently published research (Radman et al., 2022).

\subsection{Statistical analysis}

The obtained values for evaluation of antioxidant activity are expressed as mean values with standard deviations of four replicates. The differences between the means were analyzed by Tukey's test of One-Way ANOVA using GraphPad Prism 8.0 (GraphPad Software Inc., San Diego, CA, USA). Values of $p<0.05$ and lower were considered significantly different. Correlations and regression analysis describing the antioxidant activities between spectrophotometric methods (ABTS, DPPH, Folin-Ciocalteu, and ORAC assays) and voltammetric analysis (SWV) were conducted using the regression program in GraphPad Prism 8.0.

\section{Results}

\subsection{Voltammetric analysis}

In this research, square-wave voltammetry was employed to determine the antioxidant capacity of the dry residues of extracts from brown macroalgae samples immobilized on the surface of GCE and immersed into a $0.1 \mathrm{~mol} / \mathrm{L}$ phosphate buffer solution. To evaluate their activity and optimal electrochemical response, a change in the $\mathrm{pH}$ values, ranging from 3 to 11, for all obtained extracts was tested. As can be seen from Figure 1a-f, the $\mathrm{pH}$ of the solution affects the voltammetric response of each sample, i.e., the oxidation current is strongly dependent on $\mathrm{pH}$ (data not shown). The potential of the lead peak for all samples was independent over the $\mathrm{pH}$ range. Voltamograms for fraction $\mathrm{F} 2$ for both Dictyota species (Fig. 1a and 1c) have similar electrooxidative response which consists of one oxidation peak at the potentials $E_{\mathrm{P}}=0.704 \mathrm{~V}$ and $E_{\mathrm{P}}=0.748 \mathrm{~V} v \mathrm{~s}$. $\mathrm{Ag} / \mathrm{AgCl} / 3$ $\mathrm{mol} / \mathrm{L} \mathrm{KCl}$, respectively. The voltammogram of the dry residue of fraction $\mathrm{F} 2$ from the $H$. scoparia is rather different with a lead oxidation peak at the potential $E \mathrm{p} 1=0.622 \mathrm{~V}$ and a "shoulder" peak at $E_{\mathrm{P} 2}=0.960 \mathrm{~V}$ which becomes more distinctive with a higher $\mathrm{pH}$ value (Figure 1e). As can be seen from Fig. 1b,d, and f, fractions F3 for all three samples are overall less electroactive than F2 fractions. For Dictyota species, DIDI F3 and DIFA F3, the most pronounced peak was observed at the potentials $E_{\mathrm{P}}=0.878 \mathrm{~V}$ and $E_{\mathrm{P}}=0.928 \mathrm{~V}$ at $\mathrm{pH}$ 7 , respectively. Voltammogram for HASC F3 fraction is similar to voltammogram for its F2 fraction, but it can be observed that $\mathrm{pH}$-value has a higher influence on its response, with the lead oxidation peak at the potential $E_{\mathrm{P} 1}=0.471 \mathrm{~V}$ and a "shoulder" peak at $E_{\mathrm{P} 2}=$ $0.669 \mathrm{~V}$ at the most alkaline electrolyte, $\mathrm{pH} 11$.

Under alkaline experimental conditions ( $\mathrm{pH} 11)$, square-wave voltammograms were recorded by changing the frequency in the range from 10 to $200 \mathrm{~Hz}$ for all tested samples. The peak potential was highly dependent on the logarithm of frequency $(10 \mathrm{~Hz}<f<200$ $\mathrm{Hz}$ ) for all macroalgae extracts. Cyclic voltammograms of macroalgae extracts were recorded in PBS ( $\mathrm{pH} 11$ ) within the potential range from -0.5 to $1.2 \mathrm{~V}$ and at a scan rate of 50 $\mathrm{mV} / \mathrm{s}$ as a support for the electrochemical mechanism of extracts (data not shown). 

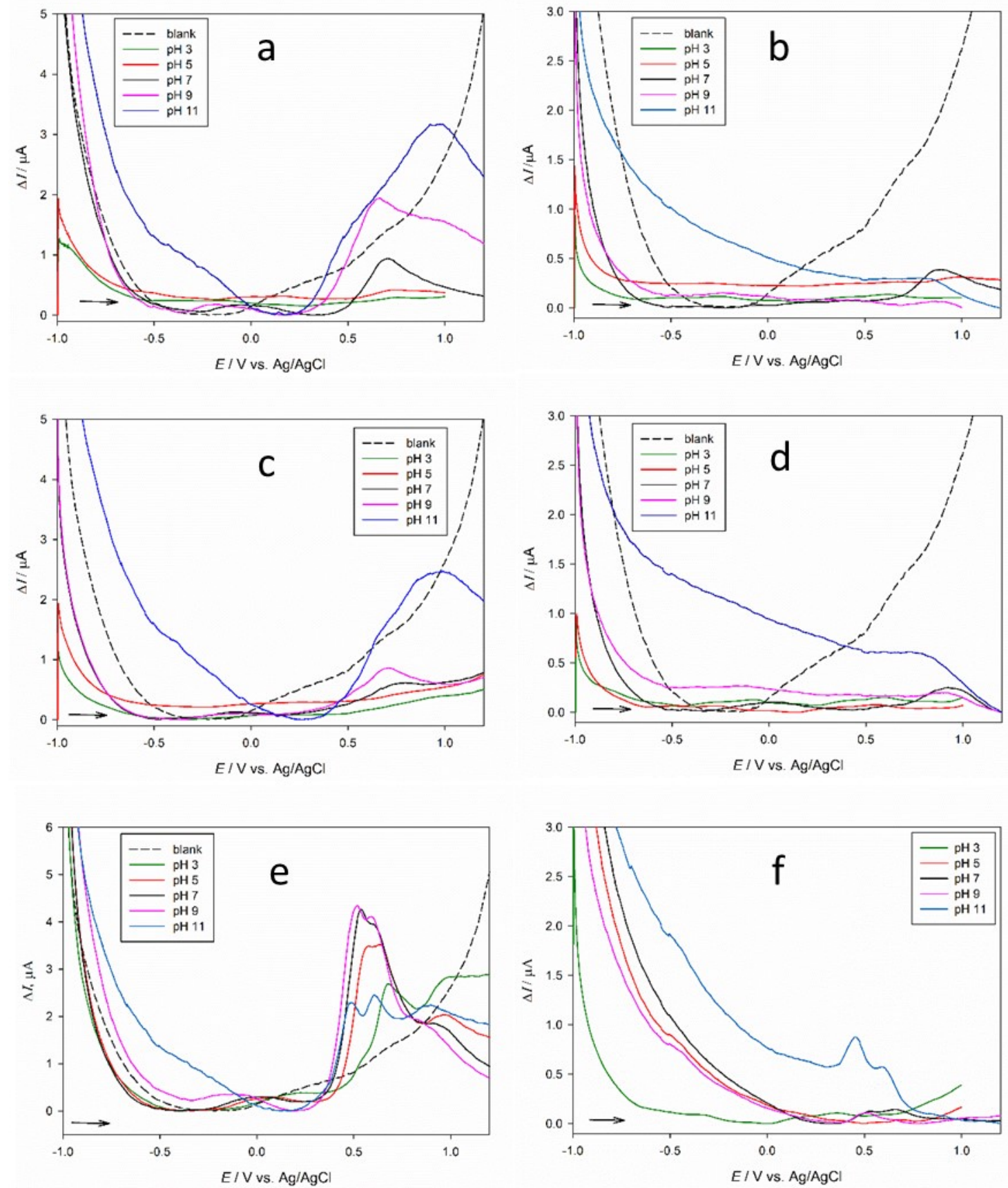

Figure 1. Square-wave voltammetric response curves obtained under different $\mathrm{pH}$ conditions for all samples: (a) Dictyota dichotoma (DIDI) F2, (b) DIDI F3, (c) Dichtyota fasciola (DIFA) F2, (d) DIFA F3, (e) Halopteris scoparia (HASC) F2, and (f) HASC F3 while immobilized on the surface of glassy-carbon electrode (GCE) and immersed in $0.1 \mathrm{M}$ PBS. The frequency was $50 \mathrm{~Hz}$, pulse amplitude was $20 \mathrm{mV}$, and the step potential was $2 \mathrm{mV}$.

To assess antioxidant activity using voltammetric analysis, the area under the curve (AUC) was integrated, the value of which represents an estimate of the total antioxidant activity of the extracts. To express total antioxidant capacity in gallic acid equivalents (GAE), a calibration was carried out in which AUC was plotted against different concentrations of the gallic acid standard $(y=581304 x-125.52)$. The highest antioxidant activity based on the AUC value was obtained for HASC F2 $(0.396 \pm 0.032 \mathrm{mg} / \mathrm{g}$ GAE), followed by HASC F3 $(0.234 \pm 0.080 \mathrm{mg} / \mathrm{g}$ GAE $)>$ DIFA F2 $(0.099 \pm 0.001 \mathrm{mg} / \mathrm{g}$ GAE $)>$ DIFA 
F3 $(0.068 \pm 0.006 \mathrm{mg} / \mathrm{g}$ GAE) $>$ DIDI F3 $(0.050 \pm 0.002 \mathrm{mg} / \mathrm{g} \mathrm{GAE})>$ DIDI F2 $(0.028 \pm 0.002 \mathrm{mg} / \mathrm{g}$ GAE). Compared to HASC F2 antioxidant activity, HASC F3 showed 1.7 folds lower activity $(p<0.001)$, while on average 7 folds lower activity $(p<0.001)$ was obtained for all fractions of Dictyota species. No significant difference in antioxidant activity was observed between fractions of two Dictyota species.

\subsection{Spectrophotometric analysis}

Four other spectrophotometric methods regarding the assessment of antioxidant activity were employed, namely ABTS, DPPH, Folin-Ciocalteu, and ORAC assays, and diverse results were obtained. As depicted in Figure $2 \mathrm{a}$, results of the DPPH assay, revealed the highest antioxidant activity for HASC F2 with the inhibition percentage around 35\% for the concentration of $5 \mathrm{mg} / \mathrm{mL}$, followed by DIDI F3 and DIFA F2 extract with inhibition percentages around $20 \%$. When normalized per gram of the fraction, HASC F2 $(9.411 \pm 2.436 \mathrm{mg}$ AAE/g fraction) showed a significantly higher $(p<0.0001)$ antioxidant activity than all other fractions.
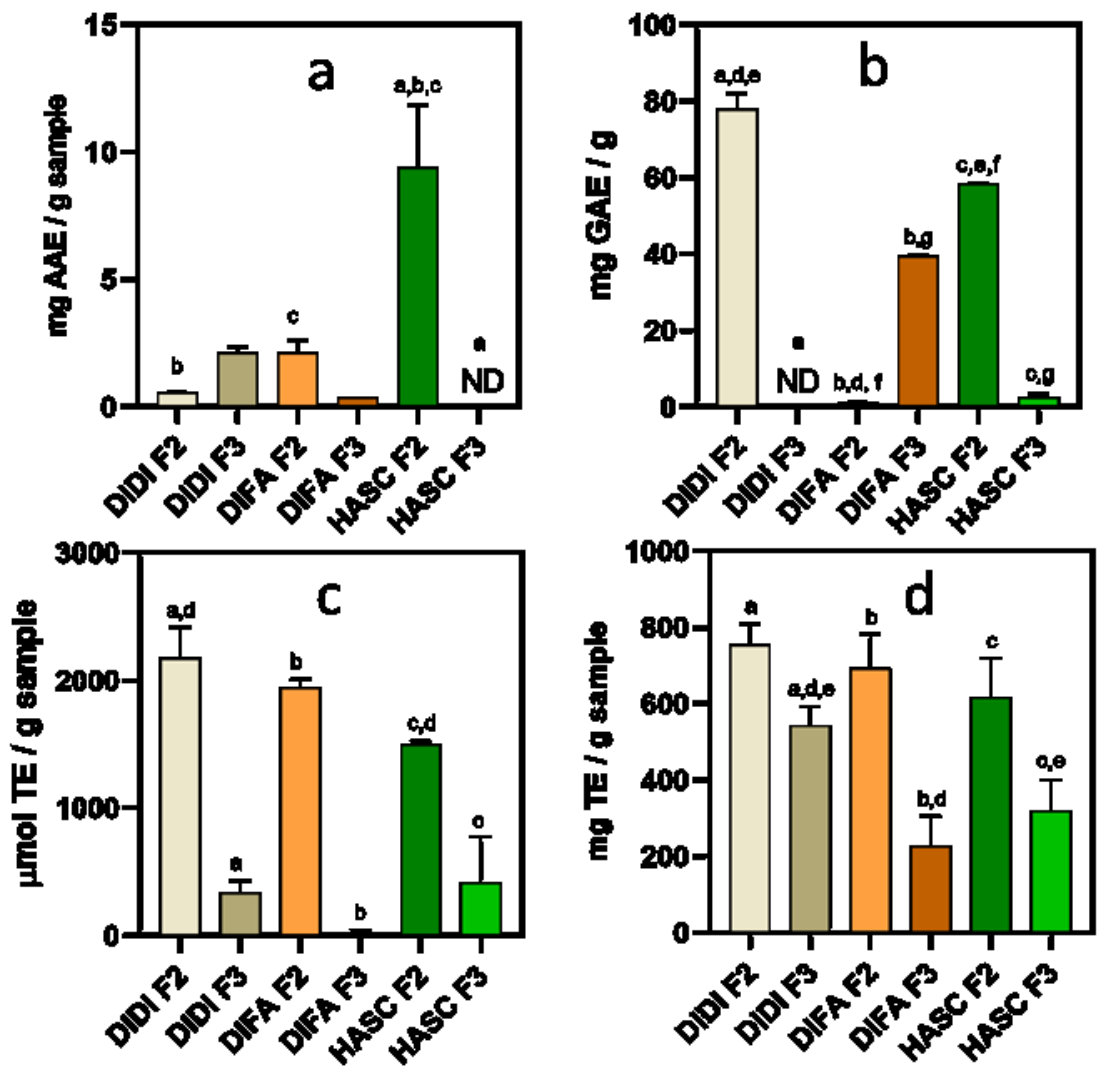

Figure 2. Radical scavenging effect of two less polar fractions from three brown macroalgae, Dictyota dichotoma (DIDI), Dichtyota fasciola (DIFA), and Halopteris scoparia (HASC), using (a) 2,2-diphenyl-1-picryl-hydrazyl (DPPH), (b) Folin-Ciocalteu (c) oxygen radical absorbance capacity (ORAC) and (d) reduction of the radical cation (ABTS) in vitro assays (mean \pm SD; $n=4)$. Columns sharing the same letters indicate a significant difference between all samples $(p<0.001)$. ND - none determined.

The results using the Folin-Ciocalteu assay revealed high activity for two F2 fractions, DIDI F2 (77.84 $\pm 4.19 \mathrm{mg} / \mathrm{g}$ GAE) and HASC F2 (58.26 $\pm 0.22 \mathrm{mg} / \mathrm{g} \mathrm{GAE})$, moderate activity for DIFA F3 (39.47 $\pm 0.52 \mathrm{mg} / \mathrm{g}$ GAE), low activity for HASC F3 $(2.36 \pm 1.12 \mathrm{mg} / \mathrm{g}$ GAE) and DIFA F2 (1.13 $\pm 0.32 \mathrm{mg} / \mathrm{g}$ GAE), while no activity was observed for DIDI F3.

Antioxidant activity of the different polarity fractions from brown macroalgae varied from 25 to $2500 \mu \mathrm{M} / \mathrm{g}$ TE by implementing the ORAC assay (Fig. 1c). Samples with a high ORAC activity were all three methanolic fractions: DIDI F2 $(2175.18 \pm 246.73 \mu \mathrm{M} / \mathrm{g}$ 
TE), DIFA F2 (1944.24 $\pm 63.95 \mu \mathrm{M} / \mathrm{g}$ TE) and HASC F2 (1497.50 $\pm 26.49 \mu \mathrm{M} / \mathrm{g}$ TE), while F3 fractions for all three samples exerted significantly lower activity $(p<0.001)$, as can be seen at Fig. 1c.

The fourth method implemented in this research was the reduction of the radical cation by implementing an ABTS assay (Fig 1d). Different concentrations of F2 and F3 for all algae were prepared in the range from 0.005 to $15 \mathrm{mg} / \mathrm{mL}$ to obtain $\mathrm{IC}_{50}$ curves. The IC 50 values for both samples were calculated as shown in Table 1, with the corresponding confidence interval, slope, and coefficient of determination $\left(\mathrm{R}^{2}\right)$. As can be seen, the lowest $\mathrm{IC}_{50}$ value, i.e., the highest antioxidant activity was obtained for F2 of HASC, followed by F2 fractions of DIDI and DIFA which exhibited almost the same activity. Interestingly, IC 50 values for fraction F3 of DIDI and DIFA could not be determined because the upper inhibition plateau could not be reached, while the $\mathrm{IC}_{50}$ value for HASC F3 is relatively high indicating lower antioxidant activity of this sample.

Table 1. Dose-inhibition results using the ABTS in vitro assay $(n=4)$ to obtain the half-maximal inhibitory concentration $\left(\mathrm{IC}_{50}\right)$ with the presented confidence intervals, Hillslope, and $\mathrm{R}^{2}$ value.

\begin{tabular}{|l|c|c|c|c|c|}
\hline \multicolumn{2}{|c|}{ Sample } & $\begin{array}{c}\text { IC }_{50} \text { value, } \\
\mathbf{m g} / \mathbf{m L}\end{array}$ & Confidence interval & Hillslope & R $^{2}$ value \\
\hline \multirow{3}{*}{ Dictyota dichotoma } & F2 & 2.595 & $1.889-2.899$ & 1.122 & 0.987 \\
\cline { 2 - 6 } & F3 & ND & - & - & - \\
\hline \multirow{2}{*}{ Dictyota fasciola } & F2 & 2.582 & $2.078-3.404$ & 1.254 & 0.984 \\
\cline { 2 - 6 } & F3 & ND & - & - & - \\
\hline \multirow{2}{*}{ Halopteris scoparia } & F2 & 1.947 & $1.332-4.320$ & 1.130 & 0.964 \\
\cline { 2 - 6 } & F3 & 4.317 & $3.065-5.102$ & 1.854 & 0.925 \\
\hline
\end{tabular}

To evaluate the correlation between electrochemical (SWV) and spectrophotometric (ABTS, DPPH, Folin-Ciocalteu, and ORAC) methods for all samples, Pearson's correlation coefficient (Utakod et al., 2017) was applied. Multiple regression analysis between SWV and DPPH showed a good correlation. The Pearson's correlation coefficient for SWV versus DPPH was a positive value of $0.83(p<0.05)$. For other spectrophotometric methods, there was no correlation obtained with SWV. Additionally, a statistically significant $(p<0.05)$ high positive correlation between ABTS and ORAC with Pearson's correlation coefficient of 0.94 was observed. A medium positive correlation between ABTS versus DPPH and Folin-Ciocalteu methods was found with Pearson's correlation coefficients of 0.35 and 0.37 , respectively. Also, a medium positive correlation between DPPH and Folin-Ciocalteu and ORAC assays was observed with Pearson's correlation coefficients of 0.29 and 0.30, respectively. Lastly, Pearson's correlation coefficient of 0.34 was obtained between results of ORAC and Folin-Ciocalteu assays.

\section{Discussion}

Numerous studies in the recent years have been focused on finding important bioactive compounds with antioxidative activity from natural sources such as marine organisms (Corsetto et al., 2020). Within this study, in vitro evaluation of the antioxidative activity of two fractions, methanolic (F2) and dichloromethanolic (F3) obtained from three brown macroalgae (DIDI, DIFA and HASC) was first performed by implementation of square-wave voltammetry (SWV) since voltammetric methods determine antioxidant activity by measuring the electrondonating capacity. This method has attracted some attention in the last decade because it provides fast and sensitive response and evaluation of antioxidant activity of extracts obtained from both macro- and microalgae samples (Goiris et al., 2012; Ragubeer et al., 2010; Ragubeer et al., 2012). However, it is still underexploited, probably due to the complex interpretation of the obtained results and the fact that extracted compounds need to be electroactive to provide a signal. In this rese- 
arch, focus was on naturally occurring organic compounds in brown macroalgae, and since they could act as antioxidants through electron transfer, it was considered that they would be largely responsible for the antioxidant activity of analyzed samples. It is known that the oxidation potentials of compounds correlate with their antioxidant activity, i.e., samples with less positive oxidation potential possess higher radical scavenging activity (Hotta et al., 2002). As can be seen from Fig. 1b,d, and f, fractions F3 for all three samples are overall less electroactive, probably because the mostly non-polar molecules with small or none electroactivity are present in that fractions. (Ir)reversibility of reactions for all sample fractions was determined by changing the frequency and plotting the first peak P1 potential with the logarithm of frequency $(10 \mathrm{~Hz}<f<200 \mathrm{~Hz})$. The linear dependence of oxidation peak potentials for all F2 and F3 fractions with the logarithm of frequency confirmed that these processes are irreversible (Mirčeski et al., 2007). Subsequently, by conducting the cyclic voltammetry $(\mathrm{CV})$ in phosphate buffer solution $(\mathrm{pH}$ 11), all macroalgae showed lower sensitivity but confirmed the (ir)reversibility of these processes for each obtained peak. Because SWV yielded more and better-defined peaks than the $\mathrm{CV}$, it was used for the determination of the antioxidant profile of these samples. The results of AUC were summarized and expressed as gallic acid equivalents for comparison with spectropohotmetric assays. Detailed electrochemical analysis of gallic acid by means of square-wave voltammetry (SWV) was performed in our recent study (Čižmek et al., 2021). The obtained results showed higher antioxidant activity for HASC F2 and F3 fractions than for DIDI and DIFA samples, i.e. most of the area that is quantified from the voltammograms of HASC has a lower oxidation potential, around $0.600 \mathrm{~V}$ (Fig. $1 \mathrm{e}, \mathrm{f})$.

Since antioxidants act by several mechanisms and a single assay cannot accurately reflect all of the antioxidants in a complex fraction (Antolovich et al., 2002; Gan et al., 2017) but also to evaluate used voltammetric method, in this research antioxidant activity was evaluated by employing additional four spectrophotometric methods. DPPH assay measures the antioxidant action via both single electron transfer (a SET mechanism) and by radical quenching (hydrogen atom transfer (HAT)), and is most commonly used method for estimation of total antioxidant activity. DPPH activity is relatively low when expressed per gram of extract for all tested fractions, while two Dictyota species showed simmilar activity, $H$. scoparia showed the highest activity, especially F2 fraction. Results of the DPPH assay for D. dichotoma are in agreement with other studies, athough the extraction and fractionation steps were different (Çelenk et al., 2016; El-Shaibany et al., 2020). When analyzing and correlating different extraction methods, El-Shaibany et al., 2020 found that the different polarity extracts showed different but low antioxidant activity, explained by complexity of their composition. Additionally, Çelenk et al., 2016 evaluated the antioxidant activities of 24 marine macroalgae, among which was $D$. dichotoma, and also observed lower antioxidant activity in the extract obtained by maceration in methanol for 10 days. Results obtained by Folin-Ciocalteu method revealed that this assay should not be considered as a measure of total phenolic content, but rather as the rate of overall antioxidant capacity, similar to ABTS assay because some nonphenolic compounds exhibit considerable reactivity toward Folin-Ciocalteu reagent (Everette et al., 2010; Sánchez-Rangel et al., 2013). Obtained results are in accordance with study evaluating six Dictyotales samples, among which were DIDI and DIFA extracted with methanol overnight (Ktari et al., 2021). Results also showed higher activity for DIDI than the DIFA sample. Implementation of ORAC and ABTS assays revealed similar antioxidant behavior of tested samples i.e. methanolic fraction of all three samples showed higher activity. Recently, Güner et al., 2019 analyzed three different extracts from $H$. scoparia, namely hexane, chloroform, and methanol extracts utilizing DPPH and ABTS assays. The results for methanol extracts were similar to the results of this study (lower activity by implanting DPPH assay, and higher inhibition percentage for ABTS assay).

The applied correlations using Pearson's correlation coefficient suggest that numerous compounds were extracted from each macroalga and consequently exhibited different modes of action, but also had a synergistic effect. Therefore, the lack of significant correlation between methods can be explained. However, this research provided infor- 
mation that SWV can be used to assess the antioxidant activity of the macroalgae samples and implies the potential usage of voltammetry to quickly determine their antioxidant activity.

\section{Conclusions}

Three brown macroalgae Dictyota dichotoma, Dictyota fasciola, and Halopteris scoparia from the Adriatic Sea (Croatia) were investigated in this study with a respect to their antioxidant activity by implementing electrochemical and spectrophotometrical measurements. Fractionation by solid-phase extraction (SPE) allowed the separation of compounds of different polarities with the focus on less polar compounds. By implementing square-wave voltammetry (SWV), lower electrooxidation potential was obtained for all methanolic fractions. The highest overall antioxidant activity was observed for methanolic fraction F2 of H. scoparia, while both Dictyota species exert similar activity indicating a similar chemical composition. Correlation between voltammetric and spectrophotometric methods showed that electrochemical analysis can be used for the determination of antioxidant activity. Overall, these findings indicate the potential of brown macroalgae as a source of beneficial antioxidant agents in the different areas of industry. However, detailed chemical analysis and determination of a leading electrooxidation mechanism are still pending and will provide additional information necessary for the elucidation of such complex systems.

Funding: The authors acknowledge the finantial support from Slovenian Research Agency (ARRS), the research core funding No. P3-0388 (Mechanisms of health maintenance). This research was supported also by the Croatian Government and the European Union (European Regional Development Fund-the Competitiveness and Cohesion Operational Program - KK.01.1.1.01) funding it through the project Bioprospecting of the Adriatic Sea (KK.01.1.1.01.0002), granted to The Scientific Centre of Excellence for Marine Bioprospecting-BioProCro. One-month financial support for postdoctoral research at the Faculty of Health Sciences at Ljubljana, Slovenia, awarded to L. Čižmek by the Institute Ruđer Bošković is gratefully acknowledged.

Conflicts of Interest: The authors declare no conflict of interest.

1. Afonso NC, Catarino MD, Silva AMS, Cardoso SM. Brown macroalgae as valuable food ingredients. Antioxidants. 2019; 8: 365. DOI:10.3390/antiox8090365.

2. Antolovich M, Prenzler PD, Patsalides E, et al. Methods for testing antioxidant activity. Analyst. 2002; 122: 11-34. DOI: 10.1039/b009171p

3. Cardoso SM, Carvalho LG, Silva PJ, Pereira L. Bioproducts from seaweeds: A review with special focus on the Iberian Peninsula. Curr. Org. Chem. 2014; 18: 896-917. DOI: 10.2174/138527281807140515154116

4. Cikoš A-M, Flanjak I, Bojanić K, et al.,. Bioprospecting of coralline red alga Amphiroa rigida J.V. Lamouroux: Volatiles, fatty acids and pigments. Molecules. 2021; 26: 520. DOI:10.3390/molecules26030520.

5. Çelenk FG, Özkaya AB, Sukatar A. Macroalgae of Izmir Gulf: Dictyotaceae exhibit high in vitro anti-cancer activity independent from their antioxidant capabilities. Cytotechnology. 2016; 68: 2667-2676 . https://doi.org/10.1007/s10616-016-9991-0

6. Corsetto PA, Montorfano G, Zava S, et al. Characterization of antioxidant potential of seaweed extracts for enrichment of convenience food. Antioxidants 2020, 9: 249. DOI: 10.3390/antiox9030249.

7. Čižmek L, Bavcon Kralj M, Čož-Rakovac R, et al.Supercritical carbon dioxide extraction of four medicinal Mediterranean plants: Investigation of chemical composition and antioxidant activity. Molecules. 2021; 26: 5697.

DOI: $10.3390 /$ molecules26185697.

8. El-Shaibany A, AL-Habori M, Al-Maqtari T, Al-Mahbashi H. The Yemeni Brown Algae Dictyota dichotoma Exhibit High In Vitro Anticancer Activity Independent of Its Antioxidant Capability. BioMed Research International. 2020; 9 pages. https://doi.org/10.1155/2020/2425693

9. Everette JD, Bryant QM, Green, AM et al. Thorough study of reactivity of various compound classes toward the Folin-Ciocalteu reagent. J. Agric. Food Chem. 2010; 58: 8139-8144. DOI: 10.1021/jf1005935.

10. Gan J, Feng Y, He Z, et al. Correlations between antioxidant activity and alkaloids and phenols of Maca (Lepidium meyenii). J. Food Qual. 2017; 2017: 1-10. DOI: 10.1155/2017/3185945.

11. Goiris K, Muylaert K, Fraeye I, et al. Antioxidant potential of microalgae in relation to their phenolic and carotenoid content. J. Appl. Phycol. 2012; 24:1477-1486. DOI 10.1007/s10811-012-9804-6. 
12. Güner A, Nalbantsoy A, Sukatar A, Yavaşoğlu NÜK. Apoptosis-inducing activities of Halopteris scoparia L. Sauvageau (Brown algae) on cancer cells and its biosafety and antioxidant properties. Cytotechnology. 2019; 71:687-704.

DOI: 10.1007/s10616-019-00314-5.

13. Haque MA, Morozova K, Ferrentino G, Scampicchio M. Electrochemical methods to evaluate the antioxidant activity and capacity of foods: A review. Electroanalysis. 2021; 33: 1419-1435. DOI: 10.1002/elan.202060600.

14. Hotta H, Nagano S, Ueda M, et al. Higher radical scavenging activities of polyphenolic antioxidants can be ascribed to chemical reactions following their oxidation. Biochim. Biophys. Acta-Gen. Subj. 2002; 1572: 123-132.

DOI: 10.1016/S0304-4165(02)00285-4.

15. Ktari L, Mdallel C, Aoun B, et al. Fucoxanthin and phenolic contents of six Dictyotales from the Tunisian coasts with an emphasis for a green extraction using a supercritical $\mathrm{CO}_{2}$ method. Front. Mar. Sci. 2021; 8: 647159.

DOI: 10.3389/fmars.2021.647159.

16. Kohen R, Nyska A. Oxidation of biological systems: oxidative stress phenomena, antioxidants, redox reactions, and methods for their quantification. Toxicol. Pathol. 2002; 30: 620-650. DOI: 10.1080/01926230290166724.

17. Mirčeski V, Komorsky-Lovrić Š, Lovrić M. Square-Wave Voltammetry: Theory and Application. 1st ed.; Springer: Heidelberg, Germany, 2007.

18. Prior RL, $\mathrm{Wu} X$, Schaich K. Standardized methods for the determination of antioxidant capacity and phenolics in foods and dietary supplements. J. Agric. Food Chem. 2005; 53: 4290-4302. DOI: 10.1021/jf0502698.

19. Rabanal M, Ponce NMA, Navarro DA, et al. The system of fucoidans from the brown seaweed Dictyota dichotoma: Chemical analysis and antiviral activity. Carb. Pol. 2014; 101: 804- 811. DOI: 10.1016/j.carbpol.2013.10.019.

20. Radman S, Čižmek L, Babić S, et al. Bioprospecting of less-polar fractions of Ericaria crinita and Ericaria amentacea: Developmental toxicity and antioxidant activity. Mar. Drugs. 2022; 20: 57. DOI: 10.3390/md20010057.

21. Ragubeer N, Beukes DR, Limson JL. Critical assessment of voltammetry for rapid screening of antioxidants in marine algae. Food Chem. 2010; 121: 227-232. DOI: 10.1016/j.foodchem.2009.11.076.

22. Ragubeer N, Limson JL, Beukes DR. Electrochemistry-guided isolation of antioxidant metabolites from Sargassum elegans. Food Chem. 2012; 131: 286-290. DOI: 10.1016/j.foodchem.2011.08.037.

23. Ruocco N, Costantini S, Guariniello S, Costantini M. Polysaccharides from the marine environment with pharmacological, cosmeceutical and nutraceutical potential. Molecules. 2016; 21:551. DOI:10.3390/molecules21050551.

24. Sánchez-Rangel JC, Benavides J, Heredia JB, et al. The Folin-Ciocalteu assay revisited: Improvement of its specificity for total phenolic content determination. Anal. Methods. 2013; 5: 5990-5999. DOI: 10.1039/c3ay41125g.

25. Sathya R, Kanaga N, Sankar P, Jeeva S. Antioxidant properties of phlorotannins from brown seaweed Cystoseira trinodis Forsskå) C. Agardh. Arab. J. Chem. 2017; 10: S2608-S2614. DOI: 10.1016/j.arabjc.2013.09.039.

26. Utakod N, Laosripaiboon W, Chunhachart O, Issakul K. The efficiency and the correlation between testing methods on antimicrobial and antioxidant activities of selected medicinal essential oils. Int. Food Res. J. 2017; 24: $2616-2624$.

27. Zhao C, Yang C, Liu B, et al. Bioactive compounds from marine macroalgae and their hypoglycemic benefits. Trends Food Sci. Technol. 2018; 72: 1-12. DOI: 10.1016/j.tifs.2017.12.001. 